\title{
Unconscious Priming: Masked Primes Facilitate Change Detection and Change Identification Performance
}

\author{
Karen Murphy ${ }^{1} \&$ Jason Andalis ${ }^{1}$ \\ ${ }^{1}$ School of Applied Psychology and Behavioural Basis of Health Program, Griffith Health Institute, Griffith \\ University, Gold Coast, QLD 4222, Australia \\ Correspondence: Karen Murphy, School of Applied Psychology, Gold Coast campus, Griffith University. Gold \\ Coast, Queensland 4222, Australia. Tel: 61-7-5552-8952. E-mail: k.murphy@griffith.edu.au
}

Received: November 22, 2012

Accepted: December 24, $2012 \quad$ Online Published: February 21, 2013

doi:10.5539/ijps.v5n1p45

URL: http://dx.doi.org/10.5539/ijps.v5n1p45

\begin{abstract}
Change blindness refers to the finding that people have difficulty detecting changes between visual scenes, when these scenes are separated by a brief interruption to visual input. The masked priming paradigm was integrated into a change detection task using real world photos to examine if unconsciously perceived words could assist in the detection and identification of changes. Results demonstrated superior detection accuracy for deletion and location changes compared to addition changes and that change detection response times were shorter for deletion than either addition or location changes. Identification of deletion and addition changes was better than for location changes. Both change detection and identification performances were enhanced by a masked identity prime presented prior to the first scene in the change detection task. These results provide evidence that unattended information can assist change detection and change identification performance.
\end{abstract}

Keywords: change blindness, change detection, change identification, priming, masked priming, unconscious priming

\section{Introduction}

People usually have extremely well developed abilities to detect changes during uninterrupted viewing of a scene. However, when these changes occur during a brief interruption of visual input people are often unable to detect even quite large changes, a phenomenon known as change blindness (Rensink, O’Regan, \& Clark, 1997; Simons, 2000; Simons \& Levin, 1998). Research has shown that change blindness occurs under a variety of circumstances including when there is a short temporal interval between scenes (e.g., Mondy \& Coltheart, 2000), during a saccadic eye movement (Grimes, 1996; McConkie \& Currie, 1996), across scenes in a motion picture (e.g., Levin \& Simons, 1997) and in real world interactions (Simons \& Levin, 1998). For reviews see Rensink (2002) and Simons and Rensink (2005).

A number of accounts have been proposed to explain the occurrence of change blindness. According to Rensink and colleagues (Rensink et al., 1997; Rensink 2002; O'Regan, Rensink, \& Clark, 1999), when there is no interval between visual scenes, any difference between two scenes is readily apparent as an abrupt visual onset attracts attention to the location of the change and therefore change blindness does not occur. However, when there is even a short temporal interval between two scenes, the onset of the new scene produces multiple abrupt visual onsets and with no unique abrupt visual onset to guide attention to the location of a change, this produces change blindness. The aim of this study was to examine if change blindness could be reduced through the use of a cue unconsciously guiding attention in a change detection and change identification task.

Mondy and Coltheart (2000) investigated change detection and change identification performance across different types of changes. They used computer administered displays of real world photographs where participants viewed the first scene, followed by a blue pattern mask, then either the original photograph or a changed version of the original photograph. Participants were asked to indicate if they thought a change had occurred across the two displays and if possible identify what the actual change was. Four types of changes (additions, deletions, colour and location) were investigated. Additions involved the adding of a new object to the scene and deletions involved removing an object from the scene. Colour changes involved the changing of an object's colour in the second scene and location changes involved movement of an object in the second scene. Mondy and Coltheart (2000) found that mean percentage correct change identification was highest for deletions 
(66\%), then additions (51\%), followed by colour changes (43\%) and lowest for location changes (31\%). Mean percentage correct change detection rates were higher overall but showed the same pattern. Detection was highest for deletions $(81 \%)$, then additions $(66 \%)$, followed by colour changes $(57 \%)$ and lowest for location changes $(53 \%)$.

These findings indicate that people retain little information across successive views of a scene and that successful change detection requires information in the first scene to be attended and transferred into visual short term memory. That is, the iconic memory trace (visual sensory memory) (Sperling, 1960) of the target in scene one needs to be transferred and consolidated in visual short term memory (Phillips, 1974; Phillips \& Baddeley, 1971) to allow comparison with scene two. Although, iconic memory capacity is large, this level of representation is subject to overwriting by new visual information (e.g., scene two) if it is not consolidated into short term memory. This overwriting of the non-consolidated target representations produces change blindness. Mondy and Coltheart (2000) explained the superior accuracy of deletions over additions, by suggesting attention and verbal encoding of an item at initial presentation, assists the survival of this information over the blank interruption. For deletions, the object can be encoded in the first scene as it is present. However, for additions, the object is not present in the initial scene and cannot be encoded into short term memory. Furthermore, verbal encoding enhanced identification of changes that can be readily labelled or described, but not changes more difficult to describe such as location changes.

The above findings and explanation are consistent with the earlier work of Agostinelli, Sherman, Fazio and Hearst (1986). These researchers proposed that change detection occurred in two stages. Firstly, there was initial awareness of the change (detection), followed by a specification of the nature of the change (identification). The change detection stage was easier as people can often report they saw something change, but cannot describe the actual item that changed. In performing a change detection/identification task a comparison process was used, whereby features in the first scene were searched for in the second scene. This strategy would work well in most change detection tasks as observers know their task is to detect changes, thereby encouraging a focus of attention on the first scene. Accordingly, deletions (the removal of an object in the second scene) should be easier to detect than additions (the addition of an object in the second scene), because the deleted feature is present as a subject of the comparisons.

Numerous studies have demonstrated that it is possible to enhance change detection performance by cueing attributes of the changed object. For example, Rensink, et al. (1997) showed that valid or partially valid verbal cues presented at the start of each trial, improved change identification relative to the invalid cue condition, and Smith and Schenk (2008) found that a sudden onset peripheral cue facilitated change detection but only when the change occurred within 150 milliseconds of the cue presentation. Other studies have examined the impact of a cue presented between scenes in a change detection task. Becker, Pashler and Anstis (2000) used a cue presented in the interval between scenes, which resulted in improved change detection accuracy. They also found that as the time between the cue and onset of the second scene increased, change detection performance improved. These results were extended by Landman, Spekreijse and Lamme (2003), who also showed that change detection improved when the change location was cued during the interval between the two scenes. However, they also determined that if the cue appeared after the onset of scene two, performance was reduced to four items, which is consistent with the capacity of visual short term memory (Coltheart, 1980; Sperling, 1960) and the overwriting explanation. Thus it appears that by attending to a specific item in iconic memory, the item could be moved to the more stable representation of visual short term memory (Phillips, 1974; Phillips \& Baddeley, 1971; Sperling, 1960). As this transfer takes time, it accounts for with the improved task performance as the time interval between the cue and presentation of the second scene is increased. Hence, the additional time allowed the changed item to be transferred to visual short term memory prior to the onset of the second scene which would overwrite the iconic memory representation.

As the cues used in the studies above were consciously perceived by participants, it would appear that attention is necessary for detecting changes. However some studies have demonstrated that change detection can occur without attention. Smilek, Eastwood and Merikle (2000) used the flicker paradigm to examine if unattended information could facilitate change detection. They used two-feature (two letters/digits changed) and three-feature changes (three letters/digits changed) in their task. The results showed that it was easier to detect the three-feature (shorter response times) than the two-feature changes and that visual search became more difficult with increasing set size. This was taken as evidence that the preattentive guidance of focal attention was more efficient in the three-feature than two-feature change conditions (Smilek et al., 2000). Hence at an unconscious level the change of three features compared to two features resulted in more efficient change detection. 
In extending previous work in this area, Fernandez-Duque and Thornton (2000, Experiment 2) examined if explicit awareness was required for change detection to occur. Using a two alternative forced choice paradigm, participants were required to choose between two possible locations of a change in displays of 8 or 16 items. Results indicated that observers were able to detect the correct location of a change and that they were able to select the changed item at above chance levels, even when they reported being unaware of the change. This suggested that the cognitive processes associated with change detection and identification were capable of signalling change outside the realm of attention. This work was replicated and extended by Laloyaux, Destrebecqz and Cleeremans (2006), who showed that change detection accuracy was higher for both aware and unaware trials with the later outcome supporting implicit processing in change detection. These behavioural studies are further supported by event-related potential evidence that has shown differences in the event-related potential correlates for change compared to no change trials (Fernandez-Duque, Grossi, Thornton, \& Neville, 2003; Kimura, Katayama, \& Ohira, 2008)

Research in other areas has shown that objects or events in the visual field can influence behaviour, even when they are not consciously perceived by the observer (e.g., Chen, 1998; Fernandez-Duque \& Thornton, 2000; Graves \& Jones, 1992; Mack \& Rock, 1998). One such area is the masked priming paradigm which has been widely researched (Forster, 1998; Forster \& Davis, 1984) and therefore, could suitably be applied to a change detection task. In an early masked priming study, Forster and Davis (1984) investigated repetition-priming effects in word recognition (lexical access). Participants saw a neutral word (forward mask $500 \mathrm{~ms}$ ), a prime word $(60 \mathrm{~ms})$, followed by the target $(500 \mathrm{~ms})$ and were required to classify the target item as either a word or nonword (lexical decision task). Response times were shorter if the target word was preceded by the same word prime (dog then DOG), compared to when the prime and target words differed (cup then DOG). Thus an overlap in prime and target identity facilitated performance compared to the unrelated prime-target condition. Whilst numerous studies have demonstrated facilitatory effects of masked primes in word recognition (e.g., Draine \& Greenwald, 1998; Perea \& Gotor, 1997), masked priming has also been examined in relation to picture naming tasks. For example, Ferrand and colleagues (Ferrand, Grainger, \& Segui, 1994; Ferrand, Humphreys, \& Segui, 1998) demonstrated that a masked identity prime (word that matched the picture name) presented prior to the picture target resulted in shorter naming times than when the prime word was unrelated to the picture. This suggested that even under brief exposure durations, lexical representations of the prime activated access to the picture name.

Sandwich masking of primes is now commonly used to prevent overt identification of the prime stimulus, by interrupting the visible persistence produced by the iconic memory trace. In this procedure, masking both precedes and follows the prime word. For example, a row of hash marks (\#\#\#\#\#\#) or random letters (FTQBGDX) are presented before and after the prime word APPLE. This technique has been used effectively by various researchers in tasks such as picture naming, lexical decision and word classification (Debner \& Jacoby, 1992; Greenwald, Draine, \& Abrams, 1996; Jacoby \& Whitehouse, 1989; Klinger, Burton, \& Pitts, 2000).

Since the presentation of a masked identity prime has been shown to facilitate picture-naming latencies, the current experiment sought to investigate if unconscious priming could reduce change blindness. This study aimed to replicate the change detection and identification findings of Mondy and Coltheart (2000), and investigate the effectiveness of a masked identity prime in a change detection task. It was predicted that for both change detection and identification, accuracy would be higher for deletion than addition and location changes as in the Mondy and Coltheart (2000) study. If masked priming facilitated change detection or identification then participants would be more accurate in the identity prime condition than in the neutral or unrelated prime conditions.

\section{Method}

\subsection{Participants}

A total of 50 undergraduate University students voluntarily completed this study in return for one hour of research course credit. Data from five of these participants were not included in the final sample due to very low overall task accuracy (e.g., $<30 \%$ ) or poor performance on the no change trials in the task. That is, they tended to respond change to all trials within the experiment indicating that they were responding in a biased fashion and not assessing each individual trial presented. The final sample consisted of forty-five ( 34 female) participants between 18 and 56 years of age $(\mathrm{M}=26.95, \mathrm{SD}=10.03)$, with English as their first language, normal colour vision (Ishihara, 1994) and self-reported normal vision or corrected to normal vision (glasses or corrective lenses worn during experiment). 


\subsection{Stimuli}

\subsubsection{Change Detection/Identification Scenes}

Each participant viewed the same set of stimuli, which consisted of 45 unique changed scenes and 20 unique filler (unchanged) scenes. Photographs were selected from freely available internet sites or scanned from magazines in jpeg format and later converted to 16-bit Bitmap file format. Scanned photographs were between $11 \mathrm{~cm} \times 8 \mathrm{~cm}$ and $20.5 \mathrm{~cm} \times 15 \mathrm{~cm}$ and changed and filler pictures were similar in content and form. Filler pictures (used for no change trials) were included so that participants had to evaluate each photograph for the occurrence of a change. These catch trials allowed for identification of participants who responded in a biased fashion (e.g., indicated a change on every trial which would result in perfect accuracy for the change detection score and zero accuracy for the no change trials). Scenes were selected so that all three types of change could be made to the same target object within the picture. Additionally, the target object had to have a distinct written label to avoid confusion with any other objects presented in the photograph.

Scenes were digitally altered using Adobe Photo Deluxe software (Adobe Corporation, 1996) to create the changed versions of the photographs. The alterations did not impair the overall quality of the scenes or the visibility of any items within the photograph. Two types of changes were made to a target object, which allowed for three types of changes to be produced. Firstly, the target object was deleted from the original scene and the object replaced by background colours matching the area surrounding the deleted item. Additions and deletions involved the same picture pair shown in reverse order. When the target object was removed from the scene and the second scene was the original, the type of change was an addition. Alternatively, when the first scene was the original and the target object had been removed from the second scene, the type of change was a deletion. The second alteration involved moving the location of a target object in the original scene, so the first picture was the original and the second scene contained the location change of the target object. Changes always involved whole objects that were unique to the scene and were not made so that the addition, deletion or movement of an object caused an existing object to be blocked or a new object to be revealed. Relative depth of the object was also preserved in the location changes. Colour changes were not examined, as they were not always appropriate for the target objects in this study. For example, the target objects often possessed a complex arrangement of hue and contrasts that could not be altered without decreasing the visual quality of the object.

\subsubsection{Masked Primes}

The masked cue word presented prior to each trial was from one of three categories. Identity primes were selected from the most common name (label) of the target object in each scene. Unrelated prime words were also nouns, with no relationship to any object in the scene. Identity and unrelated primes were matched across number of letters, number of syllables and written frequency (Kucera \& Francis, 1967). The neutral primes $(\mathrm{XXXX})$ had the same number of letters as the identity and unrelated primes for each scene.

In order to determine suitable masked priming parameters, a pilot study $(n=7)$ was undertaken to ensure prime perceptibility was limited. One important factor in this pilot was that participants were told their task was purely prime identification. Furthermore, they were told the exact location of the word (i.e. between the masks) and were encouraged to guess the word identity if they were unsure. Various prime durations were examined within the pilot study and response accuracy for all trials noted. The final masked prime parameters were a $150 \mathrm{~ms}$ forward mask(@\#@\#@\#@\#@\#@\#),34 ms prime duration (presented in uppercase font size 12) and 17 ms backward mask (\$\&@\#\$\&). This resulted in overall prime identification of $29 \%$ in the pilot study. Participants often reported poor confidence in their responses, regardless of their accuracy. This degree of accuracy was deemed sufficiently low, as two other factors would be present in the main study to limit overt identification. One was that participants would not be told about the presentation of the primes at the start of each trial and the other was that that the task demands of the change detection task would limit the time of attentional focus on the words.

\subsection{Scene, Prime and Experiment Version Matching}

As the target objects varied in salience across the pictures, a pilot experiment was run asking participants to rate the salience of each target object within each picture. The participants $(n=7)$ completing the masked priming pilot also rated the salience of each target object on a ten point likert scale. In completing this rating, they were instructed to pay attention to factors such as size of the object, complexity, centrality, distinctiveness and the number of other objects present. These results were used in equating salience across the types of change and prime conditions for the experiment proper. Refer to the following section on scene version allocation for use of these pilot data. 
Nine versions of the experiment were created to allow each scene to be shown to participants across all possible change and prime types across the course of the experiment. For example, if an object was shown as a deletion in one version of the experiment, it was shown as an addition and location change in the other versions and for each of the three change types the masked prime was presented as an identity, unrelated or neutral prime item. Each version of the experiment contained an equal number of trials for the addition, deletion, and location changes and within each change type one-third of the pictures were preceded by an identity prime, one-third an unrelated prime and one-third the neutral prime. The same filler (no change) items were used in each of the nine versions of the experiment.

To ensure comparability across the nine versions of the experiment a between version Analysis of Variance (ANOVA) was run on relevant prime word (identity and unrelated) parameters including written word frequency $(\log 10+1$ transformed), number of letters, and number of syllables. An ANOVA was also run to compare the target object salience ratings (from the above pilot study) across the nine versions of the experiment. For each of the above dependent variables there was no significant difference across the nine versions (all $p s>.05$ ). Hence all nine versions of the experiment were matched on all relevant variables.

\subsection{Apparatus and Procedure}

The experiment was run using DMDX software (Forster \& Forster, 2003), on a Dell (733 MHz) Pentium III laptop, connected to a separate $60 \mathrm{~Hz} 14$-inch UVGA monitor. A separate keyboard was connected to the laptop to record participant responses. Participants were tested at a viewing distance of approximately $50 \mathrm{~cm}$, in a dimly lit testing cubicle.

Participants were tested individually in a session lasting approximately 45 minutes (range 35 to 60 minutes to complete experiment). They firstly read the information sheet and indicated their agreement to participate by signing informed consent then provided age, handedness and gender details. Verbal and onscreen instructions were provided for participants prior to the start of the 12 practice trials. These practice trials were a combination of changed and filler (no change) pictures from the different change and prime types. None of the pictures used in the practice trials appeared in the experiment proper. The practice trials were followed by one block of 65 experimental trials, 15 trials for each of addition, deletion or location changes and 20 filler items. The DMDX program (Forster \& Forster, 2003) presented the trials in a random order for each participant.

Each trial commenced with a central fixation cross (1 second). A forward mask (@\#@\#@\#@\#@\#@\#)was then presented for $150 \mathrm{~ms}$, and the prime for $34 \mathrm{~ms}$, followed by a backward mask (\$\&@\#\$\&) for $17 \mathrm{~ms}$. Immediately following the backward mask, the first photograph was presented for four seconds. This was then replaced with a blank blue screen ( 1 second) and the second picture was presented for 4 seconds. Participants were instructed to study the pictures in as much detail as possible and to be prepared for a change (addition, deletion or location change) in any of the objects and that the second picture might or might not have been changed. Participants were then instructed to push the "CHANGE" or "NO CHANGE" button as quickly and accurately as possible, once the second scene had been compared. The "CHANGE" button (right "Shift" key) and the "NO CHANGE" button (left "Shift" key) were reversed for left-handers, so that the dominant hand always pushed the "CHANGE" button. Response times were measured from the onset of the second scene and participants were given a maximum of 10 seconds to respond change/ no change. After the second photograph, a prompt appeared on the computer screen saying, "If you saw a change, please write down what changed. Press SPACEBAR to start the next trial". This self-paced procedure enabled participants' unlimited time to record on the response sheet, a description of the type of change and the object involved or to tick the no change box. Participants were instructed to take a break between trials at any time, by waiting to push the spacebar before progressing to the next trial. At completion of the experiment, participants were asked if they noticed any of the masked prime words. This information was noted and they were then debriefed on the aims of the study.

\subsection{Design}

The dependent variables were percent correct for the change detection trials (\%) and response times (ms) to correctly detect a change within the scene. As is standard practice in experimental psychology outlying response times were replaced with values $+/-2.5$ standard deviations from the mean for each individual participant. Change identification responses were scored as correct (percentage) if the participant correctly identified the type of change and object involved in the change. The within subjects independent variables were change type (addition, deletion or location change) and prime condition (related, unrelated or neutral). 


\section{Results}

\subsection{Prime Identification}

The percentage of prime words identified was extremely low; $77 \%$ of participants reported seeing no words, $18 \%$ identified 1 to 4 words and $5 \%$ identifying 5 to 10 words. Word identification was random and was evenly dispersed across change type and prime condition. Those who identified words only reported their presence in the latter stages of the experiment and did not always connect the prime and the target object. In total, only $2.6 \%$ of words were overtly identified, which is sufficiently low to suggest explicit encoding of the prime was virtually absent in this experiment.

\subsection{Statistical Analysis}

Data for each of the dependent variables, change detection RT (ms), change detection accuracy (\% correct) and change identification accuracy (\% correct), were analysed using a 3 (change type) x 3 (prime condition) repeated measures ANOVA.

\subsection{Change Detection and Identification Performance}

Accuracy was very high for the no change filler trials $(\mathrm{M}=90.88 \%, \mathrm{SD}=8.06)$. Detection accuracy differed across the three change types, $\mathrm{F}(2,88)=8.32, \mathrm{p}<.0005, \eta \mathrm{p} 2=.16$. Pairwise comparisons revealed that detection accuracy was better for deletion and location changes than for addition changes ( $\mathrm{ps} \leq .003$ ). Refer to Table 1 for change detection and identification accuracy and change detection response time descriptive statistics. There was also a main effect of change type for the detection response times, $F(2,88)=10.29, p=<.0005, \eta p 2=.19$. Response times for detections were shorter for deletion changes compared to location and addition changes (ps $<.0005$ ). As the deletion changes resulted in both shorter response times and greater accuracy it should be noted that there is no speed-accuracy trade off in the data.

There was a difference in the change identification accuracy across the three types of changes, $\mathrm{F}(2,88)=6.65, \mathrm{p}$ $=.002, \eta \mathrm{p} 2=.13$. Pairwise comparisons revealed greater change identification accuracy for the deletion than location changes $(\mathrm{p}<.0005)$ and for addition than location changes $(\mathrm{p}=.033)$.

Table 1. Change detection and identification accuracy and detection response times by type of change

\begin{tabular}{|c|c|c|c|}
\hline & Change Detection (\%) & Change Identification (\%) & Detection Response Time (ms) \\
\hline Type of Change & Mean (SE) & Mean (SE) & Mean (SE) \\
\hline Addition & $59.41(2.21) \mathrm{a}$ & $47.58(2.25) \mathrm{a}$ & $2340(109) \mathrm{b}$ \\
\hline Deletion & $66.74(1.57) b$ & $50.92(1.80) \mathrm{a}$ & $2006(102) \mathrm{a}$ \\
\hline Location & $67.85(1.74) b$ & $42.27(1.72) \mathrm{b}$ & $2335(97) b$ \\
\hline
\end{tabular}

Note. Mean values with different superscripts are significantly different $(\mathrm{p}<.05)$ and mean values with the same superscript are not significantly different.

\subsection{Priming Change Detection and Identification}

Table 2 presents the descriptive statistics for prime condition main effects for all dependent variables. The effect of prime condition approached significance for change detection accuracy $F(2,88)=2.46, p=.083, \eta p 2=.06$, indicating a change detection advantage for targets preceded by an identity prime compared to an unrelated prime word $(\mathrm{p}=.04)$. The interaction between prime condition and change type was not significant, $F(4,176)=.65, \mathrm{p}$ $=.645, \eta \mathrm{p} 2=.01$.

Neither the main effect of prime condition, $\mathrm{F}(2,88)=.636, \mathrm{p}=.532, \eta \mathrm{p} 2=.01$, nor the interaction between prime and change type, $\mathrm{F}(4,176)=.974, \mathrm{p}=.423, \eta \mathrm{p} 2=.02$, were significant for change detection response times, indicating masked primes had no effect on the time taken to detect changes.

For change identification accuracy the effect of prime condition was significant, $F(2,88)=3.128, p=.049, \eta p 2$ $=.07$. A change identification accuracy advantage was evident for scenes preceded by an identity prime compared to those where a neutral prime was used $(\mathrm{p}=.024)$. The interaction between prime type and change type was not significant, $\mathrm{F}(4,176)=.263, \mathrm{p}=.902, \eta \mathrm{p} 2=.01$. 
Table 2. Change detection and identification accuracy and detection response times by prime condition

\begin{tabular}{llll}
\hline \multirow{2}{*}{ Prime Condition } & $\begin{array}{l}\text { Change Detection (\%) } \\
\text { Mean }(S E)\end{array}$ & $\begin{array}{l}\text { Change Identification (\%) } \\
\text { Mean }(S E)\end{array}$ & $\begin{array}{l}\text { Detection Response Time (ms) } \\
\text { Mean }(S E)\end{array}$ \\
\hline Identity & $67.85(2.02)^{\mathrm{a}}$ & $50.45(2.16)^{\mathrm{a}}$ & $2226(93)$ \\
Unrelated & $62.59(1.93)^{\mathrm{b}}$ & $46.37(2.12)^{\mathrm{a}, \mathrm{b}}$ & $2294(103)$ \\
Neutral & $63.56(1.90)^{\mathrm{a}, \mathrm{b}}$ & $43.95(1.83)^{\mathrm{b}}$ & $2221(96)$
\end{tabular}

Note. Mean values with different superscripts are significantly different $(\mathrm{p}<.05)$ and mean values with the same superscript are not significantly different.

\section{Discussion}

This study demonstrated that it was easier to detect the deletion of an object from a scene or the change in location of an object within a scene than it was to detect the addition of a new object to the second scene. The detection latency data for correct detections showed that these results were not attributable to a speed-accuracy trade-off as response times were shorter for deletion changes than location or addition changes. Identification of the changed object was more accurate for deletion and addition changes than for location changes. Importantly there was evidence of priming for both change detection and identification accuracy.

In this study there was superior change identification performance for deletion and addition changes compared to location changes. According to Mondy and Coltheart (2000), the deleted object advantage arises if participants use a verbal encoding strategy, creating a verbal list of objects present within the initial scene. This list survives the blank interruption, due to being consolidated in short term memory, and allows for a comparison of objects in the second scene. This process results in accurate identification of the missing object from scene two. In contrast, when an object is added to scene two, as it is not present in the initial scene it does not enter the list. This makes the identification of a change difficult as all the objects in the verbal list are present in scene two. It is not until the observer notices something that is present in the scene that is not in their list that they detect the object as new addition. This verbal encoding strategy would also explain the response time advantage for the detection of deleted objects compared to addition changes in this study. For example, participants would need to search their entire list before they discover a mismatch between the listed items and those presented in scene two, resulting in long response times for addition changes. In contrast, when an item is deleted this will be detected earlier as it will occur during the initial search of the listed items. Difficulty in using a verbal list strategy to encode location information in a precise way (Mondy \& Coltheart, 2000), would also account for the poorer performance for the identification of relocated objects. For example, while the list strategy would be useful for object identification this approach would have been too complex to enable the location of each object to be noted by participants. Thus participants may have noted the general location of objects (e.g., upper right quadrant) but this would not have been specific enough to be able to identify objects that moved location between the two scenes.

Compared to the results of Mondy and Coltheart (2000), participants in this experiment more readily detected location changes. This difference could be due to a greater degree of object displacement for location changes in this study compared to the location changes used by Mondy and Coltheart (2000). Alternatively, verbal encoding may not have been used reliably by all participants in the current study to aid change detection. Some may have used visual spatial working memory to store spatial location information of the initial scene (Logie, 1995), accounting for the superior location change detection performance. This may also account for the decreased change identification performance for moved objects, as identity information may not be reliably encoded in visual spatial working memory.

There was evidence to suggest that change detection performance could be facilitated by the presentation of masked prime words prior to scene one, as change detection was more accurate in the identity than unrelated prime condition. As this effect approached significance, it should be interpreted cautiously and replicated in future research. However, it is not surprising that the priming effect approached significance for change detection accuracy. For example, the prime word indicated the target object within the scene and not if a change would be present. As a result the prime was more informative for change identification accuracy than for change detection performance. This idea is supported by the superior change identification accuracy when scene one was preceded by an identity prime compared to a neutral prime. As priming occurred for identity primes compared to the unrelated prime for detection and the neutral prime for identification it could be suggested that these priming effects are different. However, both the neutral and unrelated primes were unrelated to the target object and as 
these conditions did not significantly differ from each other for either change detection or identification, this supports the occurrence of priming in the current experiment. The priming effects observed here are consistent with masked prime picture-naming tasks (Ferrand et al., 1994; Ferrand et al., 1998), which demonstrated facilitation of performance by masked primes identifying the to-be-named picture label.

The priming of change identification accuracy supports the notion that enhanced performance can occur so long as the unconsciously presented stimulus contains specific information about the changed object. This suggests that an unconscious identity prime relative to a neutral prime can facilitate the encoding of a target object within short term memory. To the authors' knowledge no studies to date have examined the use of masked priming in assisting change detection or identification. Hence this study is the first to demonstrate that it is possible to guide attention preattentively to the change in scene two through the use of primes that identify the target object.

One criticism of studies reporting unconscious priming effects is the potential contamination of conscious awareness, into what has been labelled unconscious (Fernandez-Duque, \& Thornton, 2000). In this study contamination from conscious awareness is unlikely for several reasons. Firstly, the present study used sandwich masking, plus prime durations (34 ms) briefer than Forster and Davis (1984), where they demonstrated little information was available for conscious recall of their prime words, even without a backward mask. Secondly, extensive pilot testing was undertaken to minimise overt perceptibility of prime words (in informed pilot participants). Finally, participants were questioned as to whether they noticed the prime. In total, less than $3 \%$ of all the words were overtly identified and these words were not systematically associated with one type of change or prime condition. Thus the priming effects observed in this study appear to have occurred without participant awareness.

Change detection and identification tasks have been used to examine attentional processing in different populations. For example, Yaxley and Zwaan (2005) showed that smokers' response times were shorter than non-smokers for a smoking related object change but slower to respond to a changed non-smoking object when a smoking object was in the scene. Both groups performed similarly when there was no smoking object in the scene. Non-smokers also showed this attentional bias when they were informed of the smoking focus of the task. Using a masked priming change detection task would allow further comparison of the attentional biases in these groups. For example, the presentation of a masked prime may increase the magnitude of the attentional bias in smokers and also to ascertain if it is possible to unconsciously create an attentional bias in non-smokers via the presentation of smoking related masked primes.

In summary, this study provides evidence to suggest that it is possible to enhance attentional processing of change detection and identification through the presentation of unattended target identity primes prior to scene one in a change detection task. These results further demonstrate that unattended information is important in guiding attention to changes in a change detection paradigm. The use of a masked priming change detection task in future research would further our understanding of the occurrence of change blindness.

\section{References}

Adobe Corporation. (1996). Adobe Photo Deluxe 1.0 [Computer software]. San Jose CA: Author.

Agostinelli, G., Sherman, S. J., Fazio, R. H., \& Hearst, E. S. (1986). Detecting and identifying change: Additions versus deletions. Journal of Experimental Psychology: Human Perception and Performance, 12, 445-454. http://dx.doi.org/10.1037//0096-1523.12.4.445

Becker, M. W., Pashler, H., \& Anstis, S. M. (2000). The role of iconic memory in change detection tasks. Perception, 29, 273-286.

Chen, Z. (1998). Inattentional amnesia: Evidence of perception without awareness. Investigative Ophthalmology and Visual Science, 39, 629.

Coltheart, M. (1980). Iconic memory and visible persistence. Perception and Psychophysics, 27, 183-228. http://dx.doi.org/10.3758/BF03204258

Debner, J. A., \& Jacoby, L. L. (1994). Unconscious perception: Attention awareness and control. Journal of Experimental Psychology: Learning Memory and Cognition, 20, 304-317. http://dx.doi.org/10.1037//0278-7393.20.2.304

Draine, S. C., \& Greenwald, A.G. (1998). Replicable unconscious semantic priming. Journal of Experimental Psychology: General, 127, 286-303. http://dx.doi.org/10.1037/0096-3445.127.3.286

Fernandez-Duque, D., Grossi, G., Thornton I. M., \& Neville, H. J. (2003). Representation of change: separate electrophysiological markers of attention, awareness, and implicit processing. Journal of Cognitive 
Neuroscience, 15, 491-507. http://dx.doi.org/10.1162/089892903321662895

Fernandez-Duque, D., \& Thornton, I. M. (2000). Change detection without awareness. Do explicit reports underestimate the representation of change in the visual system? Visual Cognition, 7, 323-344. http://dx.doi.org/10.1080/135062800394838

Ferrand, L., Grainger, J., \& Segui, J. (1994). A study of masked form priming in picture and word naming. Memory and Cognition, 22, 431-441. http://dx.doi.org/10.3758/BF03200868

Ferrand, L., Humphreys, G. W., \& Segui, J. (1998). Masked repetition and phonological priming in picture naming. Perception and Psychophysics, 60, 263-274. http://dx.doi.org/10.3758/BF03206035

Forster, K. I. (1998). The pros and cons of masked priming. Journal of Psycholinguistic Research, 27, 203-233. http://dx.doi.org/10.1023/A:1023202116609

Forster, K. I., \& Davis, C. (1984). Repetition priming and frequency attenuation in lexical access. Journal of Experimental Psychology: Learning, Memory and Cognition, 10, 680-698. http://dx.doi.org/10.1037//0278-7393.10.4.680

Forster, K. I., \& Forster, J. C. (2003). DMDX: A windows display program with millisecond accuracy. Behavior Research Methods, Instruments, and Computers, 35, 116-124. http://dx.doi.org/10.3758/BF03195503

Graves, R. E., \& Jones, B. S. (1992). Conscious visual perceptual awareness vs. non-conscious visual spatial localisation examined with normal subjects using possible analogues of blindsight and neglect. Cognitive Neuropsychology, 9, 487-508. http://dx.doi.org/10.1080/02643299208252069

Greenwald, A. G., Draine, S. C., \& Abrams, R. H. (1996). Three cognitive markers of unconscious semantic activation. Science, 273, 1699-1702. http://dx.doi.org/10.1126/science.273.5282.1699

Grimes, J. (1996). On the failure to detect changes in scenes across saccades. In K. Akins (Ed.), Perception (pp. 89-110). New York: Oxford University Press.

Ishihara, S. (1994). Ishihara's tests for colour-blindness. Tokyo, Japan: Kanehara and Company.

Jacoby, L. L., \& Whitehouse, K. (1989). An illusion of memory: False recognition influenced by unconscious perception. Journal of Experimental Psychology: General, 118, 126-135. http://dx.doi.org/10.1037/0096-3445.118.2.126

Kimera, M., Katayama, J., \& Ohira, H. (2008). Event-related brain potential evidence for implicit change detection: A replication of Fernandez-Duque et al. (2003). Neuroscience Letters, 448, 236-239. http://dx.doi.org/10.1016/j.neulet.2008.10.064

Klinger, M. R., Burton, P. C., \& Pitts, G. S. (2000). Mechanisms of unconscious priming: Response competition not spreading activation. Journal of Experimental Psychology: Learning Memory and Cognition, 26, 441-455. http://dx.doi.org/10.1037//0278-7393.26.2.441

Kucera, H., \& Francis, W. N. (1967). Computational analysis of present day American English. Providence: Brown University Press.

Laloyaux, C., Destrebecqz, A., \& Cleeremans, A. (2006). Implicit change identification: A replication of Fernandez-Duque \& Thornton (2003). Journal of Experimental Psychology: Human Perception and Performance, 32, 1366-1379. http://dx.doi.org/10.1037/0096-1523.32.6.1366

Landman, R., Spekreijse, H., \& Lamme, V. A. F. (2003). Large capacity storage of integrated objects before change blindness. Vision Research, 43, 149-164. http://dx.doi.org/10.1016/S0042-6989(02)00402-9

Levin, D. T., \& Simons, D. J. (1997). Failure to detect changes to attended objects in motion pictures. Psychonomic Bulletin and Review, 4, 501-506. http://dx.doi.org/10.3758/BF03214339

Logie, R. H. (1995). Visio-spatial working memory. Hove, Great Britain: Erlbaum.

Mack, A., \& Rock, I. (1998). Inattentional blindness. Cambridge, MA: MIT Press.

McConkie, G. W., \& Currie, C. B. (1996). Visible stability across saccades while viewing complex pictures. Journal of Experimental Psychology: Human Perception and Performance, 23, 563-581. http://dx.doi.org/10.1037/0096-1523.22.3.563

Mondy, S., \& Coltheart, V. (2000). Detection and identification of change in naturalistic scenes. Visual Cognition, 7, 281-296. http://dx.doi.org/10.1080/135062800394810

O’Regan, J. K., Rensink, R. A., \& Clark, J. J. (1999). Change blindness as a result of "mudsplashes". Nature, 
398, 34. http://dx.doi.org/10.1038/17953

Perea, M., \& Gotor, A. (1997). Associative and semantic priming effects occur at very short stimulus onset asynchronies in lexical decision and naming. Cognition, 62, 223-240. http://dx.doi.org/10.1016/S0010-0277(96)00782-2

Phillips, W. A. (1974). On the distinction between sensory storage and short-term visual memory. Perception and Psychophysics, 16, 283-290. http://dx.doi.org/10.3758/BF03203943

Phillips, W. A., \& Baddeley, A. (1971). Reaction time and short-term visual memory. Psychonomic Science, 22, 73-74.

Rensink, R. A. (2002). Change detection. Annual Review of Psychology, 53, 245-277. http://dx.doi.org/10.1146/annurev.psych.53.100901.135125

Rensink, R. A., O’Regan, J. K., \& Clark, J. J. (1997). To see or not to see: The need for attention to perceive $\begin{array}{llllll}\text { changes in } \quad \text { scenes. } & \text { Psychological }\end{array}$ http://dx.doi.org/10.1111/j.1467-9280.1997.tb00427.x

Simons, D. J. (2000). Current approaches to change blindness. Visual Cognition, 7, 1-15.

Simons, D. J., \& Levin, D. T. (1998). Failure to detect changes to people in real world interactions. Psychonomic Bulletin and Review, 5, 644-649. http://dx.doi.org/10.1080/135062800394658

Simons, D. J., \& Rensink, R. A. (2005). Change blindness: Past, present and future. Trends in Cognitive Sciences, 9, 16-20. http://dx.doi.org/10.1016/j.tics.2004.11.006

Smilek, D., Eastwood, J. D., \& Merikle, P. M. (2000). Does unattended information facilitate change detection? Journal of Experimental Psychology: Human Perception and Performance, 26, 480-487. http://dx.doi.org/10.1037/0096-1523.26.2.480

Smith, D. T., \& Schenk, T. (2008). Reflexive attention attenuates change blindness (but only briefly). Perception and Psychophysics, 70, 489-495. http://dx.doi.org/10.3758/PP.70.3.489

Sperling, G. (1960). The information available in brief presentations. Psychological Monographs: General and Applied, 74, 1-30. http://dx.doi.org/10.1037/h0093759

Yaxley, R. H., \& Zwaan, R. A. (2005). Attentional bias affects change detection. Psychonomic Bulletin and Review, 12, 1106-1111. http://dx.doi.org/10.3758/BF03206451 